\title{
Signal Tuning Scheme Design of Zhongshan Street in Yinchuan City
}

\author{
Mengyuan Han \\ School of Shanghai Maritime University, College of Transport and Communications, Shanghai, \\ 201306, China \\ 391269784@qq.com \\ Corresponding author: 391269784@qq.com
}

Keywords: traffic congestion, arterial coordinated control, traffic simulation, signal optimization, evaluation indicators

\begin{abstract}
With the accelerated pace of people's lives and works, traffic congestion, traffic accidents and traffic emissions have become one of the most important topics in the major cities of China in the last few years. The arterial coordination control method is one of the important methods to reduce traffic pollution, improve traffic efficiency and relieve traffic pressure. This paper will take traffic signal tuning scheme design of Zhongshan Street of Yinchuan city as the goal to design a group-based arterial coordinated control scheme. In the course of the study, the traditional algebraic method of signal coordination control theory and the traffic simulation software Synchro (Simtraffic) are used to develop the traffic control strategy for Zhongshan street, After the simulation, the results of the two schemes are compared with stops and travel time.The results show that this paper provides a feasible solution to alleviate the traffic congestion, improve traffic efficiency, reduce traffic pollution and reduce traffic accidents of Zhongshan street.
\end{abstract}

\section{Introduction}

In this paper, a coordinated control scheme based on grouping and simulation is designed based on coordinated control of green wave in trunk lines, and a traffic signal tuning scheme is designed for Zhongshan street, Yinchuan city, Ningxia hui autonomous region. The thesis carries out specific research according to the following five steps: firstly, the traffic status of Zhongshan street in Yinchuan city is analyzed, and then the diagnosis is made to find out the problem of traffic bottleneck. According to the traffic flow, parking times and travel time in the morning and evening peak and flat peak, the all-day control scheme of the street during peak and flat peak was studied and worked out by using feasible calculation methods. On the basis of the proposed control strategy, this paper evaluates the effect of traffic signal tuning, compares the tuning scheme with the original scheme through traffic simulation, and puts forward reasonable improvement Suggestions. In the process of designing the coordinated control strategy of green wave in the main line, this paper focuses on the fact that the current signal timing scheme cannot meet the actual traffic demand. Firstly, it carries out single-point optimization, and improves the signal timing based on the characteristics of the actual traffic flow, so as to achieve the matching between the designed lamp 
timing scheme and the actual traffic demand. Secondly, in view of the long journey time and excessive stopping times, the coordinated control strategy of green wave in trunk lines was selected, and the green wave scheme in trunk lines was designed based on the actual distance between intersections and the actual average driving speed. The effect comparison was measured by the number of stops, the travel time at intersections and the average speed of the sections.

\section{Literature review}

In foreign countries, Webster first proposed the parking times model with the goal of minimum parking times and the parking delay model with the goal of minimum average vehicle delay, which are closely related to the coordination control of trunk lines, in 1958. After nearly 40 years, Little and Morgan proposed the MAXBAND model based on the maximum green wave bandwidth. The classic feature of this model is that it takes into account the intersection of the two directions and specifies the corresponding compensation value so that vehicles moving at a certain speed can obtain the continuous driving right. The existing MAXBAND model establishes the first truly mixed integer linear programming model for coordinated control of main green wave based on the maximum green wave bandwidth by using the branch - defined solution method. Model based on MAXBAND, Gartner and Stamatiadis variable green wave band based on bandwidth of MULTIBAND two-way road green wave coordinate control model, this model introduced a known as "network closed loop constraints" the comprehensive development of MULTIBAND models to the urban traffic arteries of communication area coordination control in this area, the purpose is to consider all sorts of new complex influence factors, such as existing teams to empty time, left the vehicle behavior and the different bandwidth of each node is one. All of these results adopt the important method of mixed integer linear programming to maximize the green wave bandwidth of the inlet and outlet channels. The MAXBAND and MULTIBAND models have been widely used in many different road networks in North America and elsewhere. However, as they are the coordinated control methods of fixed time, they cannot flexibly and effectively adapt to different traffic conditions. The main disadvantage of MAXBAND model and MULTIBAND model is that their Settings are based on historical data rather than real-time traffic information. When traffic demand changes due to accidents or other reasons, the optimization Settings will fail.

Tian, then puts forward a road based on the technology of automatic dividing control area green wave coordinate control method, which means this method will need to plan the automatic traffic area is divided into multiple control area, the main traffic partition design for each small green wave coordinate control scheme, to adjust the adjacent two traffic area on both ends of the boundary intersection, the relative phase difference between the final can realize peak running in the morning and evening time between two or more control area green waves in one direction.

\section{Road profile of Zhongshan street}

Zhongshan street, Xingqing district, Yinchuan city, is a north-south main road that runs through the city and is also the main channel connecting Yinchuan development zone with the old city, bearing the traffic demand from south to north. This section is located in the old city district, close to the business district, intersecting with a number of north-south one-way lines, from Haibao road in the north to Nanxun street in the south, the whole section is divided into Zhongshan north street and Zhongshan south street, with a total length of about $3.4 \mathrm{~km}$. The road form of each road is triple carriageway road.

The intersections along Zhongshan street are all light-controlled intersections, and each intersection is a crossroad and mainly a single-point light-controlled signal control scheme. The optimal position of each intersection in this street is eight light-controlled intersections, and the 
signal control mode is relatively variable, resulting in a large change in the periodic flow, poor coordination, and high pressure at each intersection during peak hours. Among them, the five intersections of Beijing road - Zhongshan street to Nanxun street - Zhongshan street are one-way lines from north to south; The three intersections of Haibao road - Zhongshan street and Beijing road - Zhongshan street are two-way road.

A large number of residential living areas and commercial entertainment areas gather in the adjacent area on both sides of Zhongshan street, and the numerous traffic attraction sources and traffic generation sources generated by this result make the traffic flow characteristics of Zhongshan street all day long present the characteristics of large flow and high density. Through the investigation and analysis of traffic flow at morning peak, evening peak and flat peak, it is found that the traffic condition of the whole section of Zhongshan street is basically saturated all day, and the indicator function of channelization facilities is not clear, especially the signs and lines are blurred. Signal timing at some intersections needs to be improved in order to alleviate the delay caused by multiple stops. The phenomenon of non-mixing is relatively obvious, and the lateral interference on both sides of the road is serious. Lack of traffic organization at bus stops and non-light controlled intersections; Frequent roadside parking at large traffic hubs; These problems led to the Yinchuan Zhongshan street traffic jam problem is very difficult.

Table 1 Road conditions

\begin{tabular}{cccc}
\hline Point & $\begin{array}{c}\text { Number of } \\
\text { lanes }\end{array}$ & One lane width(m) & Distance from upstream intersection(m) \\
\hline Haibao road & Six-lane road & 3 & - \\
Shanghai road & Four-lane road & 3 & 780 \\
Beijing road & Four-lane road & 3 & 747 \\
Hubin street & Four-lane road & 3 & 471 \\
Wenhua street & Four-lane road & 3 & 212 \\
Jiefang street & Four-lane road & 3 & 322 \\
Xinhua street & Four-lane road & 3 & 384 \\
Nanxun street & Four-lane road & 3 & - \\
\hline
\end{tabular}

\subsection{Traffic problem analysis}

Referring to the overall situation of morning and evening peaks in Yinchuan city, according to the analysis of traffic flow at the intersections of Zhongshan street, the morning peak hours of this street are from 7:00 to 9:00, evening peak hours are from 17:00 to 19:00, and flat peak hours are from 9:00 to 17:00, 19:00 to 00:00 and 00:00 to 7:00.

\subsubsection{Analysis of traffic problems during rush hour}

(1) Traffic channelization is blurred, and pedestrians cross the street more, resulting in serious non-mixed traffic.

(2) During rush hour, some pedestrians do not obey traffic rules and cross the street forcibly.

(3) Zhongshan north street is a two-way road, and Beijing road, Shanghai road and Haibao road are the three roads with concentrated residential areas and shopping malls, resulting in heavy traffic congestion and vehicle interweaving during peak hours.

(4) During the peak period, many vehicles go north or west at the intersection of Beijing road and Zhongshan street and Shanghai road and Zhongshan street, resulting in a long queue of vehicles at the intersection. Vehicles entering the residential area are prone to overflow into the intersection, which seriously affects the traffic efficiency at the intersection. Not only that, Zhongshan street has 
some lane openings, leading to the difficulty for vehicles to enter the intersection, traffic efficiency is low.

(5) The current signal scheme cannot meet the actual traffic demand. On the basis of considering the general law of traffic flow, the existing signal control does not make a special signal control scheme for specific traffic needs. The traffic demand varies at some intersections in different directions at different times, and most intersections are empty, so it is difficult for a single scheme to alleviate the traffic pressure.

(6) Horizontal interference is very obvious, and overflow vehicles are obviously queued at some intersections. Zhongshan street during the morning and evening peak traffic greatly, Haibao road, Zhongshan street, north to south through the car is more, the downstream intersection import clearance of Shanghai road, Zhongshan street cross estuary green letter is small, needed badly in the intersection, are at least two lights during morning and evening peak, road on both sides of the store is more, the roadside parking was seriously into sections.

(7) Long journey time and multiple queues. Morning and evening rush hour traffic, vehicle parking in road along the route of intermittent phenomenon is very serious, part of the intersection of individual direction repeatedly queuing phenomenon occur frequently, such as lake street Zhongshan street north imported straight during morning and evening peak flow is big, affected by combined with the silver river lane lamp control intersection transverse interference, and temporary stop bus stop on the traffic interference, lead to the north needed to import, especially during the morning rush north imported straight three queuing phenomenon exists. According to the survey, many times during morning and evening rush hours, Haibao road, Zhongshan street intersection to hide and bide street - Zhongshan street intersection to the $3.4 \mathrm{~km}$, Zhongshan street, from the eight treasure way to hide and bide street intersection, the peak in the morning and evening, on average, each section of one-way trip time was 16.41 minutes and 16.56 minutes, north to south, the average number of parking is 6.33 times and 6.67 times respectively, wasted on waiting in line and deceleration of the travel time is too long.

\subsubsection{Analysis of traffic problems in flat peak}

(1) Large parking delay. The traffic flow of Zhongshan street is also relatively large during the flat peak period. Although there are few abnormal traffic conditions, vehicle intersections occur frequently, and intermittent parking of vehicles along the road is relatively obvious. According to multiple tests and statistics, the average number of stops at the eight light-controlled intersections of Zhongshan street in the whole section from north to south is about 5.67 times, which has a significant impact on the driving speed and a significant decrease in driving comfort.

(2) Test travel time is too long. According to the analysis of many tests, the average travel time of Zhongshan street flat peak from north to south is about 12 minutes, which is too long for the journey time of waiting and acceleration and deceleration.

\subsection{Traffic control strategy}

After investigation and analysis, according to the current traffic flow situation of Zhongshan street, the light-controlled intersections along Zhongshan street were divided into a control sub-area and coordinated control was carried out to design a green wave coordinated control scheme of the main line based on grouping with the effect of "slow in and fast out" to realize the rapid passage of vehicles.

\subsubsection{Traffic control strategy during rush hour}

(1) During the morning and evening rush hours of Haibao road - Zhongshan street - 
Shanghai-Zhongshan street section, the north entrance of Haibao road - Zhongshan street intersection has a large traffic flow, resulting in multiple queues at the north entrance of Shanghai-Zhongshan street intersection, which overflow to the upstream intersection, resulting in serious traffic congestion. In view of this situation, the congestion linkage control is adopted to alleviate the traffic pressure at the north entrance of Shanghai-Zhongshan street.

(2) To Beijing road and Zhongshan street Xinhua street and Zhongshan street, road conditions, signal cycle similar to traffic during the rush hours in the morning and evening are larger, the five intersection is main green wave coordinate control, make the upstream intersection traffic reaches the downstream intersection, queuing vehicles of the downstream intersection just start, reducing frequency of road parking. Especially during peak hours earlier, because from Beijing road and Zhongshan street left imports north to south, east and west imports - Zhongshan street, turn right into the lake street traffic flow is large, lead to traffic flow - Zhongshan street, north to the south lake street is larger, with Beijing road, Zhongshan street and lake street and Zhongshan street the silver river lane lamp control intersection between lateral vehicle interference is bigger, lead to come from Beijing road and Zhongshan street vehicles intercepted, downstream import ballast lake - Zhongshan street, north street, after being transverse interference vehicle removed, resulting in a large number of vehicles into the backlog of lakeside imports - Zhongshan street, north street. Based on the situation of road sections and traffic demand, coordinated control of the green wave from Beijing road - Zhongshan street to Xinhua street - Zhongshan street was carried out, and the north entrance phase of Hubin street - Zhongshan street was released in advance. To relieve the traffic pressure at the north entrance of Hubin street - Zhongshan street.

(3) During peak hours, the control sub-area adopts coordinated control of trunk lines to achieve balanced queuing, slow in and fast out, so as to relieve the central traffic pressure. Firstly, in view of the fact that the current signal scheme cannot meet the actual traffic demand, the single-point optimization is carried out, and the signal timing is improved in combination with the traffic flow characteristics, so as to reduce the vacancy at the intersection and relieve the traffic pressure. Secondly, according to the phenomenon of multiple queues at some intersections, coordinated control of congestion is adopted to equalize queues at each intersection, reduce the number of stops and relieve the queuing pressure.

\subsubsection{Traffic control strategy in flat peak}

(1)The traffic volume of Zhongshan street is moderate all day during the flat peak period, and there are multiple queues at some intersections. The whole section of Zhongshan street is divided into two sections according to road conditions, traffic flow, signal release mode and cycle similarity of each intersection: Haibao road - Zhongshan street - Shanghai-Zhongshan street section (two intersections in total), Beijing road - Zhongshan street - Xinhua street - Zhongshan street section (five intersections in total) for coordinated control.

(2) Haibao road - Zhongshan street and Shanghai road - Zhongshan street, according to the flow characteristics of traffic flow, according to different traffic needs, during the flat peak period, coordinated linkage control is adopted to improve traffic efficiency.

(3) From Beijing road - Zhongshan street to Xinhua street - Zhongshan street, one-way green wave coordinated control is implemented from north to south according to the flow characteristics of the traffic flow and different traffic needs, so as to reduce the number of stops, shorten the travel time and improve traffic efficiency. In a real sense to achieve the rapid passage of vehicles.

(4) In view of the fact that the current signal scheme cannot meet the actual traffic demand, the single-point optimization is firstly carried out to improve the signal timing according to the characteristics of traffic flow, so as to match the signal scheme with the actual traffic demand. In view of the long journey time and excessive stopping times, coordinated control of green wave in 
trunk lines is adopted, and the green wave scheme in trunk lines is designed in combination with segment distance and actual average speed. Reduce the number of stops and travel time.

\section{All-day traffic control program}

\subsection{Study on signal timing of single intersection based on Webster method}

The optimal period of single-point optimization is calculated by Webster method, and the calculation formula is:

$$
\mathrm{C}=(1.5 \times \mathrm{L}+5) /(1-\mathrm{y}) .
$$

where $\mathrm{C}$ is the optimal period, $\mathrm{L}$ is the total signal loss time, and $\mathrm{Y}$ is the maximum flow ratio of each phase.

For example,Taking into account the queue length, drivers' psychological state, road geometric characteristics, and the similarity of the number of bicycles and pedestrians at the entrance of Haibao road and Zhongshan street intersection, the saturation flow of each entrance road in coordinated direction and non-coordinated direction was determined to be $1650 \mathrm{pcu} / \mathrm{h}$. The single-lane saturation flow of the left-turn lane is $1550 \mathrm{pcu} / \mathrm{h}$; The saturation flow of single lane in the right turn lane is $1550 \mathrm{pcu} / \mathrm{h}$; Single-lane saturation flow in straight right lane is $1600 \mathrm{pcu} / \mathrm{h}$; The equivalent coefficient of right-turning vehicles going straight is 1.32 according to the number of pedestrians in conflict with right-turning vehicles (moderate). And start loss time: Ls $=3 \mathrm{~s}$, yellow light time: $A=3 \mathrm{~s}$, all red: $A R=0 \mathrm{~s}$, total signal loss time: $\mathrm{L}=4 \times(\mathrm{Ls}+\mathrm{i}-\mathrm{a})=4 \times(3+3-3)=$ $12 \mathrm{~s}$. According to the proportion of left-turn traffic volume in the total traffic volume of the inlet and its own calculation capacity, the intersection adopts a four-phase release mode.

Haibao road - Zhongshan street intersection north entrance lane is divided into one left, two straight to the right; The south entrance lane is divided into two left, three straight and two right; East entrance lane is divided into two left, three straight and one right; The west entrance lane is divided into one left and two straight right. The calculation methods of other points location are the same as above.

Table 2 Haibao road - Zhongshan street optimal cycle time

\begin{tabular}{ccccccc}
\hline Point location & \multicolumn{2}{c}{ Morning peak(s) } & \multicolumn{2}{c}{ Evening peak(s) } & \multicolumn{2}{c}{ Flat peak(s) } \\
\cline { 2 - 7 } & Cycle time & $\begin{array}{c}\text { Maximum } \\
\text { flow ratio }\end{array}$ & Cycle time & $\begin{array}{c}\text { Maximum } \\
\text { flow ratio }\end{array}$ & Cycle time & $\begin{array}{c}\text { Maximum } \\
\text { flow ratio }\end{array}$ \\
\hline Haibao road & 101 & 0.7725 & 130 & 0.8235 & 123 & 0.7637 \\
Shanghai road & 127 & 0.8180 & 128 & 0.8199 & 93 & 0.7508 \\
Beijing road & 118 & 0.8432 & 112 & 0.8334 & 109 & 0.8201 \\
Hubin street & 115 & 0.8785 & 109 & 0.8721 & 101 & 0.8614 \\
Webhua street & 109 & 0.8721 & 120 & 0.8600 & 104 & 0.8654 \\
Jiefang street & 110 & 0.8315 & 120 & 0.8458 & 100 & 0.8150 \\
Xinhua street & 120 & 0.8458 & 109 & 0.8229 & 105 & 0.8238 \\
Nanxun street & 93 & 0.8493 & 90 & 0.8446 & 80 & 0.8252 \\
\hline
\end{tabular}

\subsection{Rush-hour traffic control program}

\subsubsection{Control strategy selection}

Through the analysis of the all-day traffic volume of Zhongshan street and the research results of the current signal scheme, the spacing between the upstream and downstream intersections along 
Zhongshan street is no more than 800 meters, and the dispersion of vehicles in the road section is small. The green wave coordinated control intersection of the main road is a one-way traffic organization, and the whole road (excluding the widening gradient distance) is mostly a two-way four-lane road. Under the existing road resources, the current traffic condition can only be improved by reasonable traffic signal control. Most of the intersections have two or four phases. Therefore, after reasonable applicability analysis, the first two intersections of Zhongshan street (Haibao road Zhongshan street - Shanghai road - Zhongshan street) shall be controlled by link age, and the last five intersections (Beijing road - Zhongshan street - Xinhua street - Zhongshan street) shall be controlled by one-way green wave coordination.

\subsubsection{Green wave coordinates key parameter selection}

(1) Operating period:

With reference to the overall situation of morning and evening peak in Yinchuan city, according to the investigation and analysis of traffic flow at the intersections of Zhongshan street, the morning peak period is selected as 7:00- 9:00, and the evening peak period as 17:00-19:00.

(2) Key intersection:

In order to determine the common cycle of the key parameters of coordinated trunk control, it is necessary to determine the key intersections first. The intersection along Zhongshan street was optimized in a single point, and the intersection with the longest cycle time was selected as the key intersection. Through a single point of calculating optimal cycle timing signal control, and comprehensive consideration of factors such as traffic demand, selected Haibao road, Zhongshan road, Zhongshan street, street and Shanghai sections of Haibao road, Zhongshan street intersection, as the key linkage control intersection to choose Beijing road and Zhongshan street Xinhua street and Zhongshan road street Xinhua street and Zhongshan street intersection as a one-way green wave coordinate control of the key intersection.

(3) Common cycle:

According to the signal cycle optimization results of each intersection along the route, $130 \mathrm{~s}$ is selected as the common cycle of peak congestion linkage control based on the characteristics of morning and evening peak traffic flow. $120 \mathrm{~s}$ is selected as the common period of unidirectional green wave coordinated control; Nanxun street - Zhongshan street is a single-point optimized intersection. According to the traffic flow characteristics, traffic demand and the number of pedestrians crossing the street, 93s is selected as the signal cycle. The coordinated phase of each intersection is the first phase (straight from north to south), while the other phases are non-coordinated phases.

(4) Average design speed:

According to the multi-vehicle tracking test, due to the joint effect of various factors, the road speed at each intersection is uneven. Considering safety, speed limit and other factors, the average design speed of road sections with different control strategies needs to be set separately to meet the reality. During the morning and evening rush hours, the design speed of Zhongshan street linkage control section is $37 \mathrm{~km} / \mathrm{h}(10.4 \mathrm{~m} / \mathrm{s})$. Unidirectional green wave coordinated to control the design speed of the section to $30 \mathrm{~km} / \mathrm{h}(8.33 \mathrm{~m} / \mathrm{s})$. When the actual speed is greater than the designed speed, the vehicle will slow down or stop during driving. When the actual speed is less than the designed speed, the number of red light encounters by the vehicle will increase and the delay will increase.During the morning and evening rush hours, if the vehicle can drive on the road at the designed speed, it can pass the downstream intersection without meeting the red light, or when the vehicle reaches the end of the line at the downstream intersection, the vehicle at the end of the line just starts to drive; If the vehicle travels at a speed less than the designed speed, the design scheme will dissipate the queue in a way of lighting up in advance, so that the vehicle can pass through the 
intersection with less stops.

\subsubsection{Study on phase difference based on Webster method}

In addition to common period, green signal ratio, green wave bandwidth and belt speed, phase difference is the most important control parameter, which is the key to coordinate the upstream and downstream intersections of the whole section. On the basis of the optimal period calculated by Webster method, the phase difference between upstream and downstream intersections is calculated by numerical method.

(1) Phase and phase difference during morning peak hours

Based on the study on the timing of the morning peak signal, the green time distribution of coordinated phase and uncoordinated phase at each intersection is calculated.

Under the coordination control strategy, Haibao road, Zhongshan street with the Shanghai road, Zhongshan street intersection distance is $780 \mathrm{~m} \mathrm{~L}$, morning rush upstream and downstream design speed V for $37 \mathrm{~km} / \mathrm{h}(10.4 \mathrm{~m} / \mathrm{s})$, two-way phase restricted by constraints can't random values, according to solving the basic formula of phase difference $(\mathrm{T}=\mathrm{L} / \mathrm{V})$ to phase difference from north to south T12 = 780/10.4 = $75 \mathrm{~s}$; For two-way traffic, the sum of the upstream and downstream phase difference is equal to the common period, then the phase difference from south to north T21 = $130-75=75$ s; The bidirectional green wave bandwidth is the effective green time in the coordinated direction of Shanghai road and Zhongshan street.

Table 3 Morning peak phase timing table at Zhongshan street intersection

\begin{tabular}{|c|c|c|c|c|c|c|c|c|c|c|c|c|}
\hline \multirow[t]{2}{*}{ Point location } & \multicolumn{3}{|c|}{ Phase A(s) } & \multicolumn{3}{|c|}{ Phase B(s) } & \multicolumn{3}{|c|}{ Phase C(s) } & \multicolumn{3}{|c|}{ Phase D(s) } \\
\hline & $\mathrm{G}$ & A & $\mathrm{R}$ & G & A & $\mathrm{R}$ & G & A & $\mathrm{R}$ & $\mathrm{G}$ & A & $\mathrm{R}$ \\
\hline Haibao road & 63 & 3 & 64 & 23 & 3 & 101 & 13 & 3 & 114 & 16 & 3 & 111 \\
\hline Shanghai road & 36 & 3 & 91 & 20 & 3 & 107 & 40 & 3 & 87 & 22 & 3 & 105 \\
\hline Beijing road & 53 & 3 & 64 & 40 & 3 & 77 & 18 & 3 & 99 & - & - & - \\
\hline Hubin street & 60 & 3 & 57 & 54 & 3 & 63 & - & - & - & - & - & - \\
\hline Wenhua street & 77 & 3 & 40 & 37 & 3 & 80 & - & - & - & - & - & - \\
\hline Jiefang street & 54 & 3 & 63 & 33 & 3 & 84 & 24 & 3 & 93 & - & - & - \\
\hline Xinhua street & 43 & 3 & 74 & 31 & 3 & 86 & 37 & 3 & 80 & - & - & - \\
\hline Nanxun street & 59 & 3 & 31 & 28 & 3 & 62 & - & - & - & - & - & - \\
\hline
\end{tabular}

Under the coordinated control strategy of one-way green wave, the actual distance and design speed of each section are described above. The actual distance between upstream and downstream intersections is calculated by taking a significant number (for example, $47 \mathrm{~m}$ is substituted into the table). Since $(\mathrm{V} \times \mathrm{C}) / 2=(8.33 \times 120) / 2=500 \mathrm{~m}$ (take the significant number as 50$)$, the ideal spacing between upstream and downstream intersections is $500 \mathrm{~m}$, and interactive coordinated control can be adopted when the phase difference is half of the common cycle. When the phase difference of an intersection $1000 \mathrm{~m}$ apart is a common period, the signal control can adopt synchronous time coordination control. Take the first intersection (Beijing road - Zhongshan street) as the starting signal, then the distance from the first intersection is $\mathrm{VC} / 2, \mathrm{VC}, 3 \mathrm{VC} / 2 \ldots$, which can be combined as synchronous coordinated control or interactive coordinated control.The smaller the offset between the actual position and the ideal position, the better the coordinated control effect. Take $50 \pm 10$ as the appropriate range of $\mathrm{VC} / 2$, mark column a (range of ideal spacing) in the numerical solution table, then calculate the row Numbers in column a. arrange the displacement of the actual signal position and the ideal signal position in order from small to large, and count the one with the greatest difference into column b. Finally, the calculation table of relevant parameters of green wave belt was completed. 
Table 4 Distance between actual position and ideal position

\begin{tabular}{lccccc}
\hline A & 47 & 21 & 32 & 38 & b \\
\hline 40 & 7 & 28 & 20 & 18 & 12 \\
41 & 6 & 27 & 18 & 15 & 14 \\
42 & 5 & 26 & 16 & 12 & 16 \\
43 & 4 & 25 & 14 & 9 & 18 \\
44 & 3 & 24 & 12 & 6 & 20 \\
45 & 2 & 23 & 10 & 3 & 22 \\
46 & 1 & 22 & 8 & 0 & 24 \\
47 & 0 & 21 & 6 & 44 & 23 \\
48 & 47 & 20 & 4 & 42 & 22 \\
49 & 47 & 19 & 2 & 40 & 21 \\
50 & 47 & 18 & 0 & 38 & 10 \\
51 & 47 & 17 & 49 & 36 & 18 \\
52 & 47 & 16 & 48 & 34 & 17 \\
53 & 47 & 15 & 47 & 32 & 16 \\
54 & 47 & 14 & 46 & 30 & 18 \\
55 & 47 & 13 & 45 & 28 & 19 \\
56 & 47 & 12 & 44 & 26 & 20 \\
57 & 47 & 11 & 43 & 24 & 21 \\
58 & 47 & 10 & 42 & 22 & 22 \\
59 & 47 & 9 & 41 & 20 & 18 \\
60 & 47 & 8 & 40 & & \\
\hline
\end{tabular}

It can be seen from the table that, when a is 46 , b reaches the maximum value of 24 , and the offset between the ideal position and the actual position is the minimum, which can obtain a better wire control effect. Draw the distance between the ideal position and the actual position. The maximum deviation between the ideal position and the intersection of Wenhua street and Zhongshan street is $(\mathrm{a}-\mathrm{b}) / 2=(46-24) / 2=11$, that is, the deviation distance is $110 \mathrm{~m}$. Then draw the actual position and the ideal position map in proportion.

The relevant parameters of green wave belt at each intersection are calculated, and the green signal ratio (as \% of the common period) is listed in the fourth row of the table. The green loss time (the ratio of actual spacing to ideal spacing) caused by the amount of movement between the actual location and the ideal location is included in the fifth row of the table. Include the effective green letter ratio (the difference between green letter ratio and green light loss) in row 6 of the table. The upstream and downstream intersections sharing an ideal signal location adopt synchronous coordinated control, while other intersections adopt interactive coordinated control. Where, the phase difference between the actual signal position corresponding to the odd ideal signal position is 100-0.5 $\lambda \%$; The phase difference of the actual signal position corresponding to the ideal signal position of an even number is 50-0.5 $\lambda \%$, and the result is listed in the seventh row of the table. The product of the common period and the percentage of phase difference is the specific phase difference, which is listed in the eighth row of the table.

The minimum effective green signal ratio to the left of the ideal location is $12 \%$, and the minimum effective green signal ratio to the right of the ideal location is $40 \%$. The average value of the two is $26 \%$, so the green wave bandwidth during the morning peak of this scheme is $26 \%$ (31.2s). Keeping the initial selected duration of the common cycle unchanged, the belt velocity of the green wave belt should be adjusted as follows: $28 \mathrm{~km} / \mathrm{h}(7.67 \mathrm{~m} / \mathrm{s})$ according to the calculation results. 
Table 5 Calculation table of related parameters of one-way green wave belt of morning peak

\begin{tabular}{cccccc}
\hline Point location & Beijing road & Hubin road & $\begin{array}{c}\text { Wenhua } \\
\text { street }\end{array}$ & $\begin{array}{c}\text { Jiefang } \\
\text { street }\end{array}$ & $\begin{array}{c}\text { Xinhua } \\
\text { street }\end{array}$ \\
\hline Ideal position number & 1 & 2 & 2 & 3 & 4 \\
Left or right & Left & Left & Right & Left & Left \\
Green-time-rate $\lambda(\%)$ & 50 & 53 & 64 & 45 & 36 \\
Green loss time(\%) & 24 & 22 & 24 & 7 & 24 \\
Effective green ratio(\%) & 26 & 31 & 40 & 38 & 12 \\
Phase difference(\%) & 75 & 23.5 & 18 & 77.5 & 32 \\
Phase difference(s) & 90 & 28.2 & 21.6 & 93 & 38.4 \\
\hline
\end{tabular}

(2) Phase and phase difference during evening peak hours

Based on the study on the timing of evening peak signal at single intersection, the green time distribution of coordinated phase and uncoordinated phase at each intersection is calculated, as shown in table 4.28 .

Under the coordinated control strategy, the design speed of the intersection between Haibao road and Zhongshan street and Shanghai road and Zhongshan street during the evening rush hour is the same as that of the common cycle and the morning rush hour. Therefore, the phase difference T12 from north to south is $75 \mathrm{~s}$, and the phase difference T21 from south to north is $75 \mathrm{~s}$. The bidirectional green wave bandwidth is the effective green time in the coordinated direction of Shanghai road and Zhongshan street.

During the evening peak, the coordinated control of green wave in the trunk line has the same common cycle and design speed as the morning peak, so the calculation table of related parameters in the green wave belt during the evening peak is the same as that in the morning peak. See table 4.26 for the calculation process, and then the calculation table of related parameters in the green wave belt during the evening peak is completed.

Table 6 Evening peak phase timing table at Zhongshan street intersection

\begin{tabular}{cccccccccccccc}
\hline Point location & \multicolumn{3}{c}{ Phase A(s) } & \multicolumn{3}{c}{ Phase B(s) } & \multicolumn{3}{c}{ Phase C(s) } & \multicolumn{3}{c}{ Phase D(s) } \\
\cline { 2 - 14 } & G & A & R & G & A & R & G & A & R & G & A & R \\
\hline Haibao road & 43 & 3 & 84 & 29 & 3 & 98 & 22 & 3 & 105 & 24 & 3 & 107 \\
Shanghai road & 32 & 3 & 95 & 31 & 3 & 96 & 25 & 3 & 102 & 28 & 3 & 99 \\
Beijing road & 48 & 3 & 69 & 37 & 3 & 80 & 26 & 3 & 91 & - & - & - \\
Hubin street & 64 & 3 & 53 & 50 & 3 & 67 & - & - & - & - & - & - \\
Wenhua street & 73 & 3 & 44 & 41 & 3 & 76 & - & - & - & - & - & - \\
Jiefang street & 50 & 3 & 67 & 26 & 3 & 91 & 35 & 3 & 82 & - & - & - \\
Xinhua street & 59 & 3 & 58 & 28 & 3 & 89 & 24 & 3 & 93 & - & - & - \\
Nanxun street & 58 & 3 & 32 & 29 & 3 & 61 & - & - & - & - & - & - \\
\hline
\end{tabular}

Table 7 Calculation table of related parameters of one-way green wave belt of evening peak

\begin{tabular}{cccccc}
\hline Point location & Beijing road & Hubin road & $\begin{array}{c}\text { Wenhua } \\
\text { street }\end{array}$ & Jiefang street & Xinhua street \\
\hline Ideal position number & 1 & 2 & 2 & 3 & 4 \\
Left or right & Left & Left & Right & Left & Left \\
Green-time-rate $\lambda(\%)$ & 40 & 53 & 61 & 42 & 49 \\
Green loss time(\%) & 24 & 22 & 24 & 7 & 24 \\
Effective green ratio(\%) & 16 & 31 & 37 & 35 & 25 \\
Phase difference(\%) & 80 & 23.5 & 19.5 & 79 & 25.5 \\
Phase difference(s) & 96 & 28.2 & 23.4 & 94.8 & 30.6 \\
\hline
\end{tabular}


The minimum effective green signal ratio to the left of the ideal location is $16 \%$, and the minimum effective green signal ratio to the right of the ideal location is $37 \%$. The average value of the two is $34.5 \%$. Therefore, the green wave bandwidth during the evening peak of this scheme is $26.5 \%$ (31.8s). Keeping the initial selected duration of the common cycle unchanged, the belt velocity of the green wave belt should be adjusted as follows: $28 \mathrm{~km} / \mathrm{h}(7.67 \mathrm{~m} / \mathrm{s})$ according to the calculation results.

\subsection{Flat peak period traffic control program}

\subsubsection{Green wave coordinates key parameter selection}

(1) Control strategy:

Haibao road - Zhongshan street - shangshang hai road - Zhongshan street adopts congestion linkage control; Beijing road - Zhongshan street to Xinhua street - Zhongshan street adopts one-way green wave control.

(2) Operating period:

Referring to the overall situation of flat peak in Yinchuan city, according to the investigation and analysis of traffic flow at the intersections of Zhongshan street, the flat peak period is selected as 9:00-17:00, 1900-00:00 and 0000-7:00.

(3) Key intersection:

In order to determine the common cycle of the key parameters of coordinated trunk control, it is necessary to determine the key intersections first. The intersection along Zhongshan street was optimized in a single point, and the intersection with the longest cycle time was selected as the key intersection. Through a single point of calculating optimal cycle timing signal control, were selected Haibao road, Zhongshan road, Zhongshan street, street and Shanghai sections of Haibao road, Zhongshan street intersection, as the key linkage control intersection to choose Beijing road and Zhongshan street Xinhua street and Zhongshan road street Xinhua street and Zhongshan street intersection as the key part of one-way green wave intersection.

(4) Common cycle:

According to the signal cycle optimization results of each intersection along the route, $123 \mathrm{~s}$ is selected as the common cycle of flat peak congestion linkage control. $105 \mathrm{~s}$ is selected as the common period of unidirectional green wave coordinated control. Nanxun street - Zhongshan street is a single-point optimized intersection. According to the characteristics of traffic flow, traffic demand and the number of pedestrians crossing the street, 93s is selected as the signal period.

(5) Average design speed:

According to the multi-vehicle tracking test, due to the joint effect of various factors, the road speed at each intersection is uneven. Considering safety, speed limit and other factors, the average design speed of road sections with different control strategies needs to be set separately to meet the reality. During the flat peak period, the design speed of Zhongshan street linkage control section was $40 \mathrm{~km} / \mathrm{h}(11.11 \mathrm{~m} / \mathrm{s})$, and the one-way green wave control section design speed was $35 \mathrm{~km} / \mathrm{h}$ $(9.72 \mathrm{~m} / \mathrm{s})$. When the actual speed is greater than the designed speed, the vehicle will slow down or stop during driving. When the actual speed is less than the designed speed, the number of red light encounters by the vehicle will increase and the delay will increase.

\subsubsection{Study on phase difference based on Webster method}

Based on the study on the timing of morning peak signal at single intersection, the green time distribution of coordinated phase and uncoordinated phase at each intersection is calculated, as shown in the following table: 
Table 8 Flat peak phase timing table at Zhongshan street intersection

\begin{tabular}{cccccccccccccc}
\hline Point location & \multicolumn{3}{c}{ Phase A(s) } & \multicolumn{3}{c}{ Phase B(s) } & \multicolumn{3}{c}{ Phase C(s) } & \multicolumn{3}{c}{ Phase D(s) } \\
\cline { 2 - 13 } & G & A & R & G & A & R & G & A & R & G & A & R \\
\hline Haibao road & 38 & 3 & 83 & 33 & 3 & 87 & 21 & 3 & 99 & 19 & 3 & 101 \\
Shanghai road & 52 & 3 & 68 & 28 & 3 & 92 & 16 & 3 & 104 & 15 & 3 & 105 \\
Beijing road & 37 & 3 & 65 & 36 & 3 & 66 & 23 & 3 & 79 & - & - & - \\
Hubin street & 50 & 3 & 52 & 49 & 3 & 53 & - & - & - & - & - & - \\
Wenhua street & 60 & 3 & 42 & 39 & 3 & 63 & - & - & - & - & - & - \\
Jiefang street & 41 & 3 & 61 & 31 & 3 & 71 & 24 & 3 & 78 & - & - & - \\
Xinhua street & 37 & 3 & 65 & 27 & 3 & 75 & 32 & 3 & 70 & - & - & - \\
Nanxun street & 60 & 3 & 30 & 27 & 3 & 63 & - & - & - & - & - & - \\
\hline
\end{tabular}

Under the coordination control strategy, Haibao road, Zhongshan street with the Shanghai road, Zhongshan street intersection distance is $780 \mathrm{~m} \mathrm{~L}$, morning rush downstream design velocity $\mathrm{V}$ is $40 \mathrm{~km} / \mathrm{h}(11.11 \mathrm{~m} / \mathrm{s})$, two-way phase restricted by constraints can't random values, according to solving the basic formula of phase difference $(\mathrm{T}=\mathrm{L} / \mathrm{V})$ to phase difference from north to south $\mathrm{T} 12$ $=780 / 11.11=70 \mathrm{~s}$; For two-way traffic, the sum of the upstream and downstream phase difference is equal to the common period, so the phase difference from south to north T21 $=123-70=53 \mathrm{~s}$; The green wave bandwidth from north to south is the effective green time of coordinated directions of Shanghai road and Zhongshan street, and the green wave bandwidth from south to north is $24 \mathrm{~s}$.

Under the arterial coordination control strategy, because $(\mathrm{V} * \mathrm{C}) / 2=(9.72 \times 105) / 2=510 \mathrm{~m}$ (take effective number of 51), so the intersection of ideal span of $510 \mathrm{~m}, 51+10$ as appropriate range of $\mathrm{VC} / 2$, the actual location and ideal location spacing table like morning rush, look-up table 4.26, when a is 46 , maximum $24 \mathrm{~b}$, ideal position and actual position spacing figure are the same as in figure 4.9, the ideal position offset and cultural street - Zhongshan street intersection distance is $110 \mathrm{~m}$ final three.

Table 9 Calculation table of related parameters of one-way green wave belt of flat peak

\begin{tabular}{cccccc}
\hline Point location & Beijing road & Hubin road & $\begin{array}{c}\text { Wenhua } \\
\text { street }\end{array}$ & $\begin{array}{c}\text { Jiefang } \\
\text { street }\end{array}$ & $\begin{array}{c}\text { Xinhua } \\
\text { street }\end{array}$ \\
\hline Ideal position number & 1 & 2 & 2 & 3 & 4 \\
Left or right & Left & Left & Right & Left & Left \\
Green-time-rate $\lambda(\%)$ & 35 & 48 & 57 & 42 & 35 \\
Green loss time(\%) & 24 & 22 & 24 & 7 & 24 \\
Effective green ratio(\%) & 11 & 26 & 33 & 32 & 11 \\
Phase difference(\%) & 82.5 & 26 & 21.5 & 80.5 & 32.5 \\
Phase difference(s) & 86.6 & 27.3 & 22.6 & 84.5 & 34.1 \\
\hline
\end{tabular}

The minimum effective green signal ratio to the left of the ideal location is $35 \%$, and the minimum effective green signal ratio to the right of the ideal location is $57 \%$. The average value of the two is $46 \%$. Therefore, the bandwidth of the green wave during the flat peak period of this scheme is $46 \%$. Keeping the initial selected duration of the common cycle unchanged, the belt velocity of the green wave belt needs to be adjusted as: $32 \mathrm{~km} / \mathrm{h}(8.76 \mathrm{~m} / \mathrm{s})$ according to the calculated results.

\section{Conclusion}

The traffic status of urban main roads has a decisive impact on the social order and economic development of the central city. The coordinated control of green wave in Zhongshan street of 
Yinchuan city not only brings comfortable driving experience to the citizens, but also speeds up the commercial development of the city center, which is of positive significance to improving the environmental quality and reducing the accident rate of Yinchuan city. In this paper, based on the coordinated control method of green wave in the trunk line, the coordinated control scheme of green wave in the trunk line of Zhongshan street in Yinchuan city is proposed. The main conclusions are as follows:

(1) Firstly, the traffic flow characteristics and traffic flow conditions of intersections and sections along Zhongshan street at different times of the day were analyzed, the traffic lesions of this street were deeply diagnosed, and peak and flat peak traffic control strategies were formulated.

(2) The singlepoint optimization of signal timing based on Webster was completed by using the survey data of traffic volume, original signal scheme and road geometric characteristics, etc. Key parameters of trunk line coordination were set according to the optimized channelization facilities, signal cycle and phase sequence, and the design scheme of green wave belt was improved.

Through comparative analysis, the tuning scheme is better than the original scheme, and all the evaluation indexes are improved during the peak and flat peak periods. Since the idea of the tuning scheme in this paper is to make the signal control of Zhongshan street generally present an effect of "slow in and fast out", and the phase of each intersection is simple and the spacing is not large, the optimization degree shown by the comparison data is not significant, but the overall effect is good.

\section{References}

[1] Xiangjie Kong, Guojiang Shen*, Feng Xia, Chuang Lin. Urban Arterial Traffic Two-direction Green Wave Intelligent Coordination Control Technique and Its Application

[2] International Journal of Control, Automation, and System. 2011. 9(1): 60-68

[3] Jun Z. Traffic Signal Coordination Control of City Arterial Road[J]. Journal of Wuhan University of Technology (Transportation Science \& Engineering). 2011. 